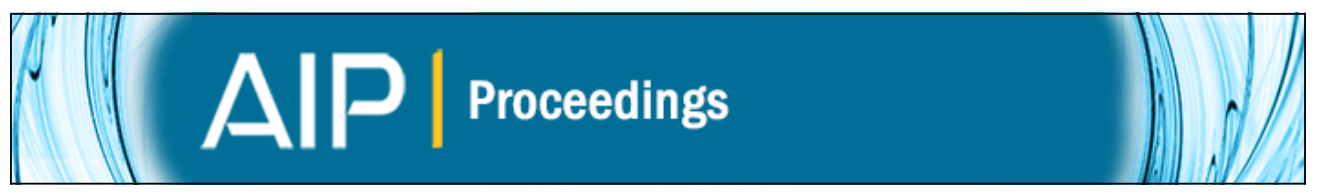

\title{
Convection: A Seismological Perspective
}

M. H. Montgomery

Citation: AIP Conference Proceedings 948, 99 (2007); doi: 10.1063/1.2819017

View online: http://dx.doi.org/10.1063/1.2819017

View Table of Contents: http://scitation.aip.org/content/aip/proceeding/aipcp/948?ver=pdfcov

Published by the AIP Publishing

Articles you may be interested in

Asteroseismology of Main-sequence Stars: Convection and Overshooting, Diffusion and Extra-mixing

AIP Conf. Proc. 948, 3 (2007); 10.1063/1.2819000

Astreroseismological results for the star V351 Ori

AIP Conf. Proc. 895, 224 (2007); 10.1063/1.2720426

Inversion method in stellar pulsation

AIP Conf. Proc. 895, 219 (2007); 10.1063/1.2720425

Radial Pulsation of the Cooling White Dwarf in the Decay of Nova Cassiopeiae 1995 (V723 Cas)

AIP Conf. Proc. 637, 311 (2002); 10.1063/1.1518221

Onset of Convection on a Pre-Runaway White Dwarf

AIP Conf. Proc. 637, 139 (2002); 10.1063/1.1518191 


\title{
Convection: A Seismological Perspective
}

\author{
M. H. Montgomery \\ Department of Astronomy, University of Texas, Austin, TX, 78712, USA
}

\begin{abstract}
I present an overview of the close relationship which convection and stellar pulsation share. This includes the driving, damping, and modulation of oscillations in stars such as the Sun, other solar-type stars, the "classical" pulsators (e.g., Cepheids, RR Lyrae), as well as more recent results from the realm of pulsating white dwarf stars.
\end{abstract}

Keywords: convection - Sun: oscillations - Sun: general - white dwarfs

PACS: $97.10 . \mathrm{Sj}$

\section{OVERVIEW}

A nearly ubiquitous presence at stellar pulsation meetings over the last several years is the "pulsational HR diagram" of Christensen-Dalsgaard (2004), shown here in Figure 1. The point of this figure is that pulsation is a common occurrence throughout the HR diagram, so by examining the pulsators we can probe a good sample of the stellar types. This allows us to test the physics in our models (and the stars!) across the HR diagram.

Convection often goes hand in hand with pulsation, for reasons which we will see are pretty obvious, so these pulsators offer us an additional window into the workings of convection in these stars, whether it be core or surface convection.

Since we will be discussing stellar pulsations, let us begin with a brief review of the relevant physics.

\section{PRIMER ON STELLAR PULSATION}

Stellar pulsations are global, normal mode oscillations of a star. For a star (or model) having spherical symmetry, the relevant pulsation quantities can be separated into a function of radius multiplied by a spherical harmonic. For example, the relative Lagrangian pressure variation can be expressed as

$$
\frac{\delta P}{P} \propto f_{n}(r) Y_{\ell m}(\boldsymbol{\theta}, \phi),
$$

where $f_{n}(r)$ is the radial function, $Y_{\ell m}$ is the spherical harmonic, $n$ is the radial "overtone number" (number of nodes), $\ell$ is the degree, and $m$ is the order of the oscillation. ${ }^{1}$

In a local analysis, there are two quantities of interest: the acoustic frequency and the buoyancy frequency. The acoustic frequency, which we denote by $S_{\ell}$, is given by

\footnotetext{
${ }^{1}$ Similarly, in Quantum Mechanics, $\ell$ corresponds to the total angular momentum while $m$ refers to the $z$-component of that angular momentum.
} 


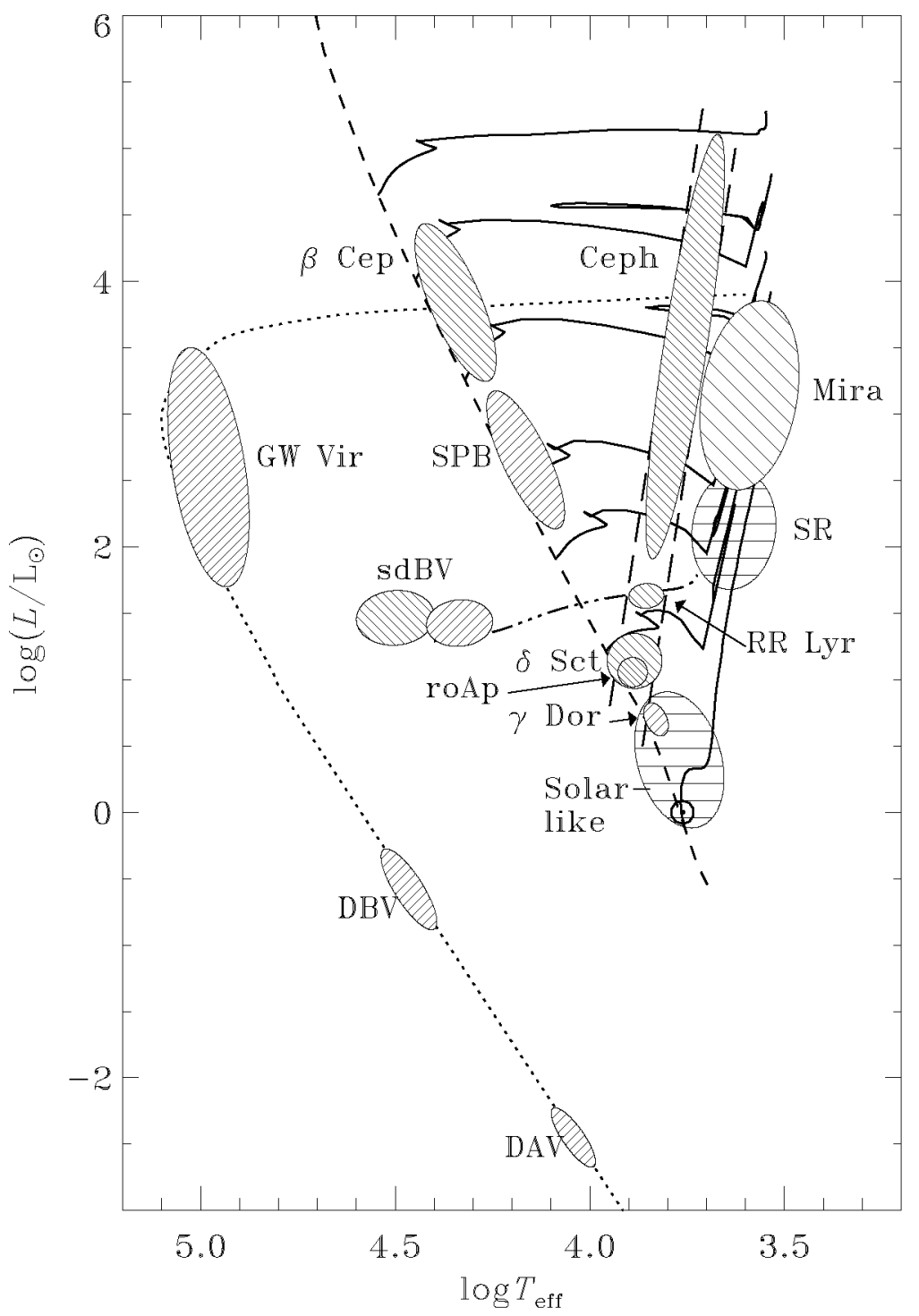

FIGURE 1. The pulsational HR diagram of Christensen-Dalsgaard (2004). Regions which are hatched with lines with $\mathrm{a}+45^{\circ}$ and $-45^{\circ}$ slope are g-mode and p-mode pulsators which are linearly unstable, respectively, either due to the $\kappa$-mechanism or convective driving. Those having horizontal hatches are solar-type pulsators, which are linearly stable but stochastically driven by their convection zones. 
$S_{\ell}^{2}=\ell(\ell+1) c^{2} / r^{2}$, where $c$ is the adiabatic sound speed and $r$ is the radius. The buoyancy frequency, $N$, is defined by

$$
N^{2}=g\left(\frac{1}{\Gamma_{1}} \frac{d \ln P}{d r}-\frac{d \ln \rho}{d r}\right),
$$

where $\Gamma_{1} \equiv(\partial \ln P / \partial \ln \rho)_{\text {ad }}$, and $P$ and $\rho$ are the pressure and density, respectively. The familiar Schwarzschild criterion for convective instability is given by the condition $N^{2}<0$.

For non-radial oscillations, there are essentially two classes of modes: high-frequency and low-frequency modes:

- $\mathrm{p}$-modes

- Pressure is the dominant restoring force.

- They are "high-frequency": $\omega^{2}>S_{\ell}^{2}, N^{2}$

- g-modes

- Gravity/buoyancy is the dominant restoring force.

- They are "low-frequency": $\omega^{2}<S_{\ell}^{2}, N^{2}$

If the local mode wavelength is much smaller than the typical scale of variations of the background state, then we can make the geometric optics approximation and talk about the path of an individual ray. In Figure 2 we illustrate a number of different ray paths characteristic of $\mathrm{p}$-modes. Rays having large values of $\ell$ have inner turning points closer to the surface, while those with small values of $\ell$ have deeper inner turning points.

When perturbations to the gravitational potential are neglected (the "Cowling approximation", Cowling 1941), the oscillation equations are reduced to a second order system, and several formulations of this system are possible (e.g. Tassoul 1980; Gough 1993). For high radial overtone number $n$, asymptotic solutions of these equations are possible.

For high-order p-modes, the frequencies become nearly evenly spaced and take on the approximate form

$$
v_{n \ell} \simeq \underbrace{\left(n+\frac{\ell}{2}+\frac{1}{4}+\alpha\right) \Delta v}_{\text {large }}-\underbrace{\left(A L^{2}-\delta\right) \frac{\Delta v^{2}}{v_{n \ell}}}_{\text {small }}, \quad \text { where } \quad \Delta v=2 \int_{0}^{R} \frac{d r}{c},
$$

and where I have indicated that the first term is much larger than the second term in this equation. Based on this relation, seismologists define the following quantities:

- "Large frequency separation": $\Delta v_{n, \ell} \equiv v_{n+1, \ell}-v_{n, \ell}$

- "Small frequency separation": $\Delta v_{n, \ell} \equiv v_{n, \ell}-v_{n-1, \ell+2}$

These quantities can be defined both from data and from the models, providing an important point of contact between the two. This pattern has been verified in detail for the case of the Sun, a p-mode pulsator.

For high-order g-modes, it is the periods which become evenly spaced, approaching the approximate relation

$$
P_{n \ell}=\frac{n}{\sqrt{\ell(\ell+1)}} \Delta \Pi, \quad \text { where } \quad \Delta \Pi \equiv 2 \pi^{2}\left(\int_{r 1}^{r 2} r^{-1} N d r\right)^{-1} .
$$




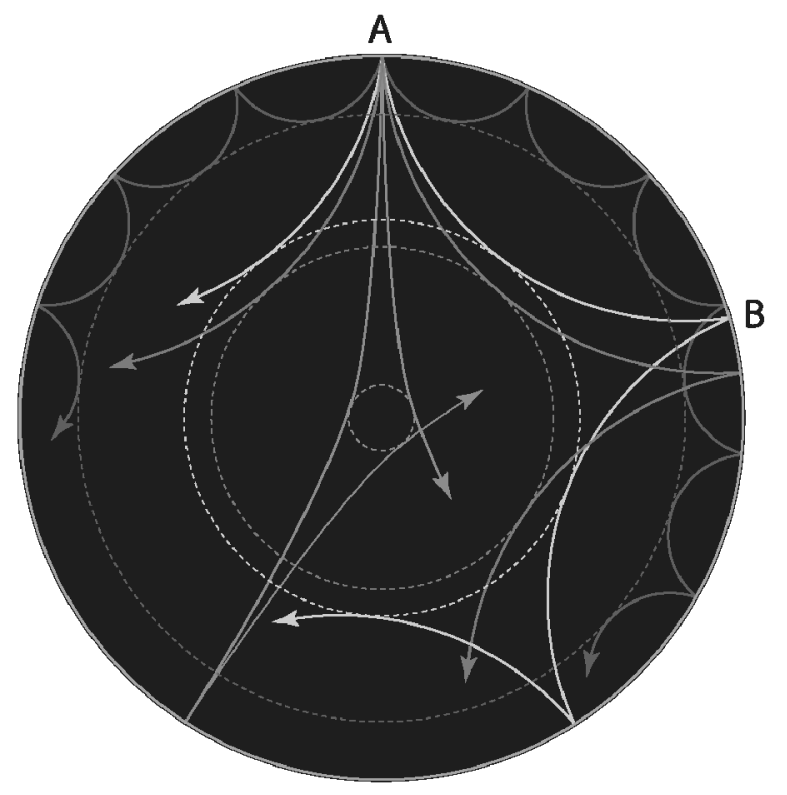

FIGURE 2. Individual ray paths of p-mode propagation.

This equal period spacing, as well as its $\ell$ dependence, has also been verified for the case of pulsating white dwarfs, which are g-mode pulsators (e.g. Winget et al. 1991, 1994).

\section{THE SOLAR CONSTRAINT}

Given the proximity of the Sun and the successes of helioseismology over the last 25 years, it is not surprising that the best single constraint for the depth of a stellar convection zone is that of the Sun (e.g. Christensen-Dalsgaard et al. 1991; Basu \& Antia 1997).

In particular, Christensen-Dalsgaard et al. (1991) provide a thorough examination of this question. Their insight was to use the extremely adiabatic structure of the solar convection zone. They defined the quantity

$$
W \equiv \frac{r^{2}}{G m} \frac{d c^{2}}{d r}
$$

which, in an adiabatically stratified convection zone having $P \sim \rho^{\Gamma_{1}}$, is equal to $W=$ $-\Gamma_{1}\left(1-\Gamma_{1}^{-1}\right)$. Thus, in the convection zone, where $\Gamma_{1} \approx 5 / 3$ one expects $W=-2 / 3$; below the convection zone $W$ should have larger values. Summarizing,

$$
W=\left\{\begin{array}{cc}
-\frac{2}{3} & \text { in convection zone } \\
>-0.4 & \text { below convection zone } .
\end{array}\right.
$$



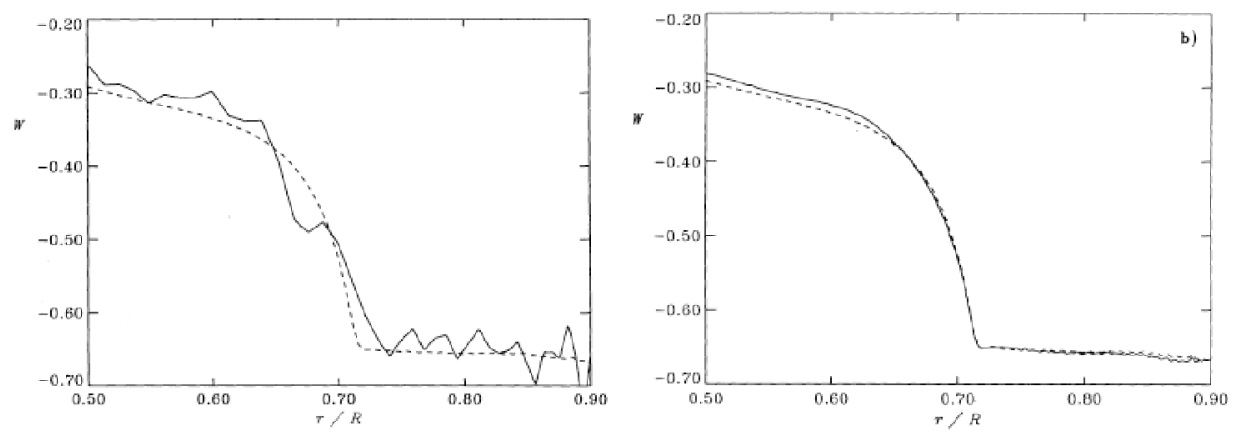

FIGURE 3. Left panel: $W$ as a function of radius as determined from the absolute method in which no reference Solar model is needed. Right panel: $W$ as a function of radius from the differential method. This method gives a depth of the convection zone as $0.287 \pm 0.003 \mathrm{R}_{\odot}$. The dashed lines are $W$ as computed in a reference Solar model. These plots are taken from Christensen-Dalsgaard et al. (1991).

The sound speed is a quantity derivable from seismology, so this constitutes a completely seismological determination of the depth of the solar convection zone.

In Figure 3 we show their result. The left panel shows $W$ as obtained from their absolute method, which only uses the observed frequencies and does not require a reference model. The right panel shows the result of using their differential method, which does require a reference model. Using this method, Christensen-Dalsgaard et al. (1991) obtain a convection zone thickness of $0.287 \pm 0.003 \mathrm{R}_{\odot}$.

\section{EXCITATION AND DAMPING OF SOLAR-TYPE OSCILLATIONS}

Far from being a bane, convection is actually a blessing for Solar-type oscillations. Basically, the small scale convective motions are thought to "shake" the star, which then rings with a certain decay time. Although the modes are linearly stable (i.e., damped), they are continuously "rung" by the convection zone, and so have a finite but small amplitude.

Why is this such a good thing? These convective motions generate "noise" with a broad spectrum of temporal and spatial scales, and this noise leads to the excitation of modes with frequencies between 1000 and $4500 \mu \mathrm{Hz}$, and $\ell$ values from zero to more than a thousand. Unlike the classical pulsators, in which only one or two modes may be excited to visible amplitudes, in Solar-type stars all the modes in a given frequency range are excited, for all values of $n$ and $\ell$. This simplifies the process of seeing the frequency patterns and making mode identifications.

Mathematically, this can also be stated simply. The individual modes can be thought of as a system of uncoupled, damped harmonic oscillators, and the stochastic driving of 


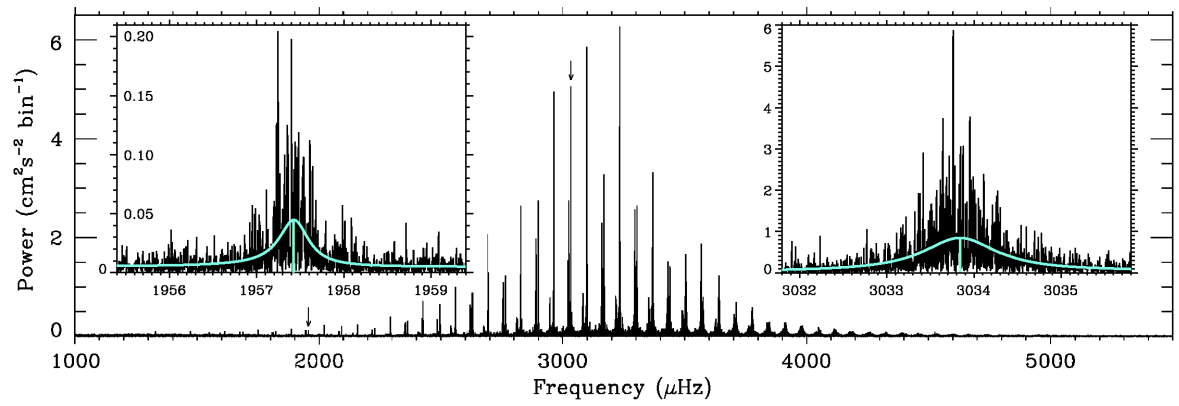

FIGURE 4. The power spectrum of BISON data taken between 1991 and 2000. The insets are closeups of the individual mode peaks showing the Lorentzian fits to these peaks.

the convection zone can be modelled as an external driving force:

$$
I_{n \ell}\left[\frac{d^{2} \xi_{n \ell}}{d t^{2}}+\Gamma_{n \ell} \frac{d \xi_{n \ell}}{d t}+\omega_{n \ell}^{2} \xi_{n \ell}\right]=f(t) .
$$

Here $I_{n \ell}$ is the "mode inertia", $\xi_{n \ell}$ is the amplitude, $\Gamma_{n \ell}$ is the damping rate, $\omega_{n \ell}$ is the natural frequency of the mode, and $f(t)$ is the external driving due to the convection zone. It is straightforward to show that the resulting power of all the oscillators in this system is

$$
P=P_{f} \sum_{n, \ell} \frac{\Gamma_{n l} / 2 \pi}{\left(\omega-\omega_{n l}\right)^{2}+\Gamma_{n l}^{2} / 4},
$$

where $P_{f}$ is the power delivered by the convection zone at a given frequency. An example of this is shown in Figure 4, which is based on data collected by BISON ${ }^{2}$ between 1991 and 2000 (Chaplin et al. 2005). A broad envelope of power between 2000 and 4000 $\mu \mathrm{Hz}$ can be seen, and the individual peaks correspond to the natural frequencies of low$\ell$ oscillation modes.

\subsection{Line Widths}

From the Lorentzian fits to the peaks, one not only obtains the mode frequencies but also their line widths, which are a measure of the intrinsic damping a mode experiences. The calculation of the damping is difficult, and it depends sensitively on the assumptions made concerning the interaction of the pulsations with the convective motions. In order to do the problem properly, one would require a time-dependent theory of convection which actually works, something which is lacking.

In place of this, several approximate approaches have been developed to address this problem. The quantities one needs to know are the perturbation to the Reynolds stresses

\footnotetext{
2 the BIrmingham Solar Oscillations Network
} 

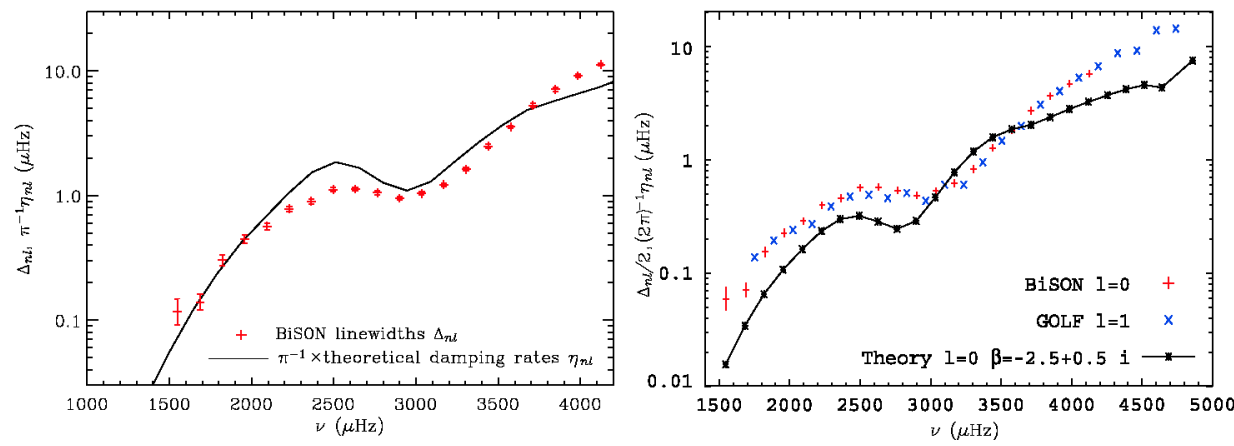

FIGURE 5. A comparison of theoretical and observed line widths as a function of frequency using the models of Houdek et al. (1999, left panel) and Dupret et al. (2004, right panel).

(e.g., the "turbulent pressure") and to the convective heat flux. One approach has been adopted by Gough (1977), and extended by Balmforth (1992a,b) and Houdek et al. (1999), and it finds that the Reynolds stresses form the more important contribution to the damping. Recently, a separate approach to the problem has been made by Dupret et al. (2004); they find that perturbations to the convective heat flux make the dominant contribution to mode stability.

The results of both sets of calculations are shown in Figure 5, along with the observed line widths of Solar p-modes: the data are the individual points and the models are the solid curves. While both models actually fit the observations fairly well, from 2000 to $3000 \mu \mathrm{Hz}$ the Houdek et al. (1999) model over-predicts whereas the Dupret et al. (2004) model under-predicts the line widths.

\section{SOLAR-TYPE STARS}

As we saw in Section 2, the large and small separations, $\Delta v$ and $\delta v$, respectively, may be useful for characterizing p-mode oscillations in the asymptotic limit. While these quantities have yet to be determined for stars other than the Sun, they will be one of the first products of the higher-precision observations which will be required for producing seismologically useful data.

Making no attempt here at complete literature search, I mention here a calculation by Monteiro et al. (2002). They made what is commonly known as a "C-D" diagram-a plot of the "small" versus the "large" separations-for stars of different masses. They found that for a given initial chemical composition and mixing length parameter $\alpha$ that models are uniquely positioned in this diagram, i.e., the position can be used as a diagnostic of the star's mass and evolutionary state. However, uncertainties in the composition and in convection blur this somewhat. In Figure 6, we show how the C-D diagram is shifted when the convective parameter is varied from $\alpha=1.833$ (dotted lines) to 1.5 (solid lines, from Monteiro et al. 2002). 


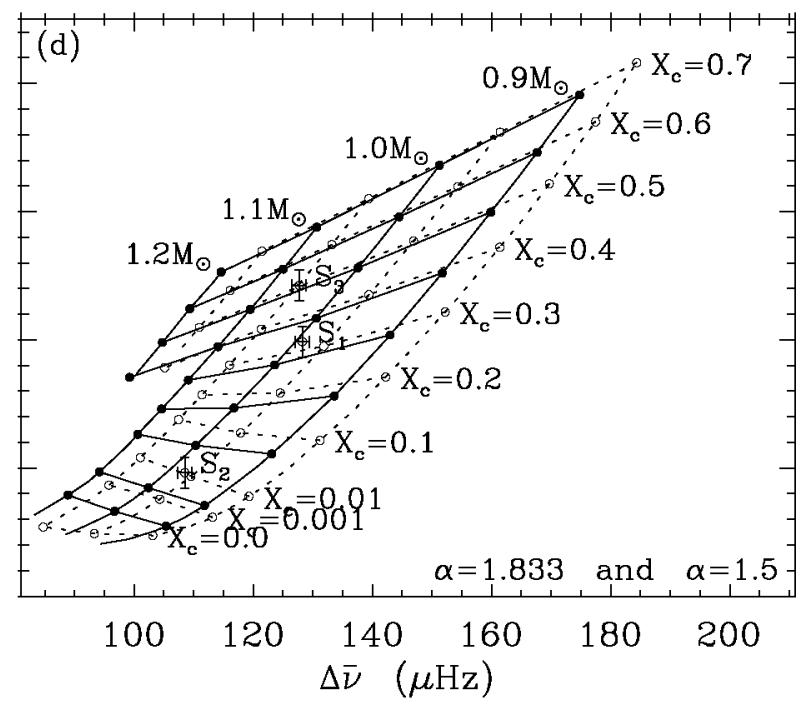

FIGURE 6. The C-D diagram for evolutionary sequences of $0.9,1.0,1.1$ and $1.2 \mathrm{M}_{\odot}$ stars - all the models have $Z=0.02, X_{0}=0.693$ (diagram from Monteiro et al. 2002). The continuous line is for models with $\alpha=1.5$ and the dotted is for the "standard" diagram with $\alpha=1.833$.

\section{THE ASSOCIATION OF CONVECTION AND PULSATION}

Pulsations in the "Clasical Instability Strip" are typically due to the " $\kappa$ mechanism", although convection is usually also present in these objects and may play some role. Simplistically, the reason for this is that in order for the $\kappa$ mechanism to provide driving there needs to be a local bump in opacity, and if the bump is large enough then convection may be favored over radiative transfer.

More precisely, it can be shown that a necessary condition for local driving of the pulsations due to the $\kappa$ mechanism is

$$
\frac{d}{d r}\left[\kappa_{T}+\kappa_{\rho} /\left(\Gamma_{3}-1\right)\right]>0,
$$

where $\kappa_{T} \equiv(\partial \ln \kappa / \partial \ln T)_{\rho}$ and $\kappa_{\rho} \equiv(\partial \ln \kappa / \partial \ln \rho)_{T}$, and $r$ is the radial coordinate. We illustrate this in Figure 7 for a "Delta Scuti-like" model. In this model, two distinct driving regions exist. The first is deeper and has a thermal timescale of $\sim 4$ hours; it is due to the second ionization of He. We see in the top panel that the convective flux is a small percentage of the overall flux, although convection is present. The second driving region is closer to the surface and has a thermal timescale of $\sim 6$ minutes; it is due to $\mathrm{H}$ ionization and the first ionization of $\mathrm{He}$. In this region we see that virtually all of the flux is carried by convection. Thus, while this region is formally a driving region for the $\kappa$ mechanism, in reality it is irrelevant; the relevant physics for driving will depend on the time-dependent interaction of the pulsations and convection. It is ironic that in many 


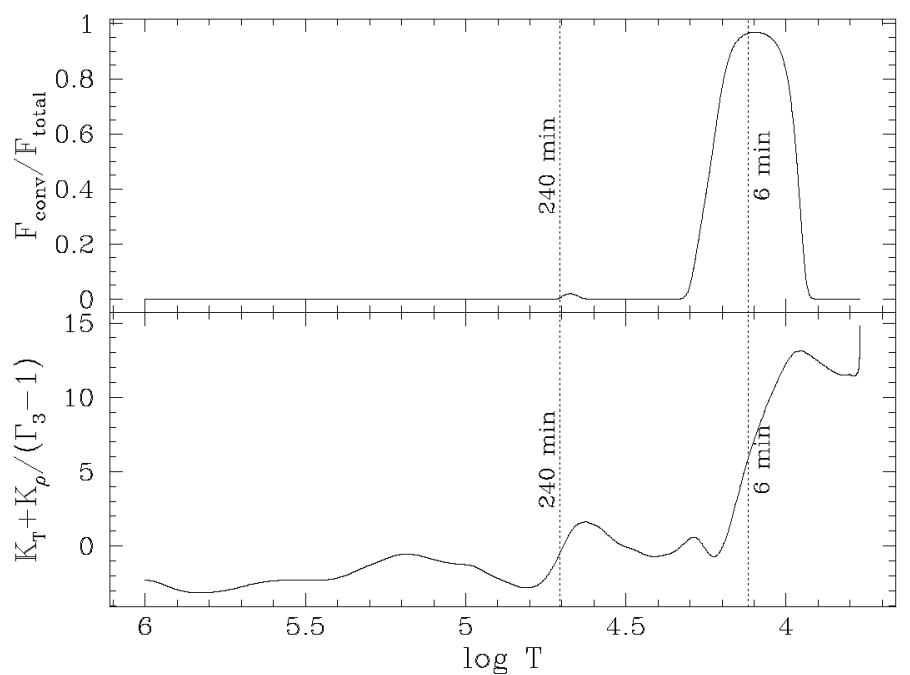

FIGURE 7. An example of the coincidence of $\kappa$ mechanism driving and convection in a Delta Scutilike model $\left(M=1.65 \mathrm{M}_{\odot}, T_{\text {eff }}=7000 \mathrm{~K}\right)$. The top panel is the fraction of the flux carried by convection, and the lower panel is the diagnostic of local driving given by Equation 9 .

instances when we expect the $\kappa$ mechanism to operate it is rendered irrelevant by the presence of convection!

\section{CONVECTION IN WHITE DWARF STARS}

The cautions of the previous section in describing the applicability of the $\kappa$ mechanism have always plagued calculations of driving and damping in white dwarfs: in all stellar models which suggest that $g$-modes are driven by the $\kappa$ mechanism, the convective flux in the driving region is quite large, usually over $90 \%$ of the total heat flux. Thus, these calculations have always been viewed with a degree of skepticism, even though they have led to reasonable predictions of the location of the DA and DB instability strips (e.g. Winget et al. 1982; Winget 1982).

In a seminal series of papers from the early 1990s, Brickhill (1991) was the first to show that the thermal interaction of the pulsations with the convection zone should actually lead to driving of the pulsations. While intriguing, his work was not widely accepted because it predicted a similar location for the instability strips, and because it was primarily a numerical investigation which was difficult to grasp. Later, Wu (1997) and Goldreich \& Wu (1999) developed an analytical approach to the problem which was essentially equivalent to Brickhill's. Their conclusions were similar, and the idea of convective driving began to be more accepted. 


\subsection{Probing Convection with Pulsations}

It was also Brickhill's original insight that the nonlinearities observed in white-dwarf pulsations should be primarily generated in the convection zone. Wu (2001) found this as well, and showed that the nonlinearities could in principle be used to constrain convection. However, her approach calculated only the lowest order nonlinearities.

Recently, Montgomery (2005a) showed that an approach combining analytical and numerical solutions could be employed, and that this would automatically include all the higher-order nonlinear terms. The assumptions of this model are listed briefly below:

1. The flux perturbations beneath the convection zone are sinusoidal in time and have the angular dependence of a spherical harmonic.

2. The convection zone is so thin that we may locally ignore the angular variation of the nonradial pulsations, i.e., we treat the pulsations locally as if they were radial.

3. The convective turnover timescale is so short compared to the pulsation periods that the convection zone can be taken to respond "instantaneously".

4. Only flux and temperature variations are considered, i.e., the large-scale fluid motions associated with the pulsations are ignored.

While item \#4 above might seem restrictive, simple mixing-length theories of convection predict that the thermal response timescale of the convection zone to perturbations in the flux incident at its base, $\tau_{\mathrm{C}}$, should be

$$
\tau_{\mathrm{C}}=\tau_{0}\left(\frac{T_{\mathrm{eff}}}{T_{0}}\right)^{-N},
$$

where $T_{\text {eff }}$ is the instantaneous effective temperature, $T_{0}$ is its time average, and $\tau_{0}$ is the time average of the convective response timescale $\tau_{\mathrm{C}}$. The exponent $N$ has a value of $\sim 90$ for the DAVs (H atmosphere WDs) and $\sim 25$ for the DBVs (He atmosphere WDs). This high power of $T_{\text {eff }}$ means that other nonlinear processes may well be negligible in comparison. These assumptions lead to the following equation relating the fluxes:

$$
F_{\mathrm{ph}}=F_{\text {base }}+\tau_{\mathrm{C}} \frac{d F_{\mathrm{ph}}}{d t}
$$

where $F_{\text {base }}=A \operatorname{Re}\left[e^{i \omega t} Y_{\ell m}(\theta, \phi)\right]$ is the flux incident at the base of the convection zone, $F_{\text {ph }}$ is the flux which emerges from the top of the convection zone in the photosphere, and $\tau_{\mathrm{C}}$ is the instantaneous thermal response timescale, which is a function of $T_{\text {eff }}$ and therefore $F_{\mathrm{ph}}$. By varying $A, \tau_{0}, N$, and the inclination angle $\theta_{\mathrm{i}}$, the calculated light curves can be fit to the observations.

This has been done for several different stars (Montgomery 2005a,b, 2006, 2007). We illustrate this in Figure 8 for the star PG1351+489, a DBV, and in Figure 9 for the star G29-38, a DAV. These stars represent the best possible targets, since at the time of the observations each was nearly mono-periodic and had a moderate-to-large amplitude of $\sim 5 \%$ in the fractional intensity.

Table 1 provides a summary of the parameters derived from the light curve fits, along with estimates of the uncertainties. Of particular note are the fits to two different epochs 


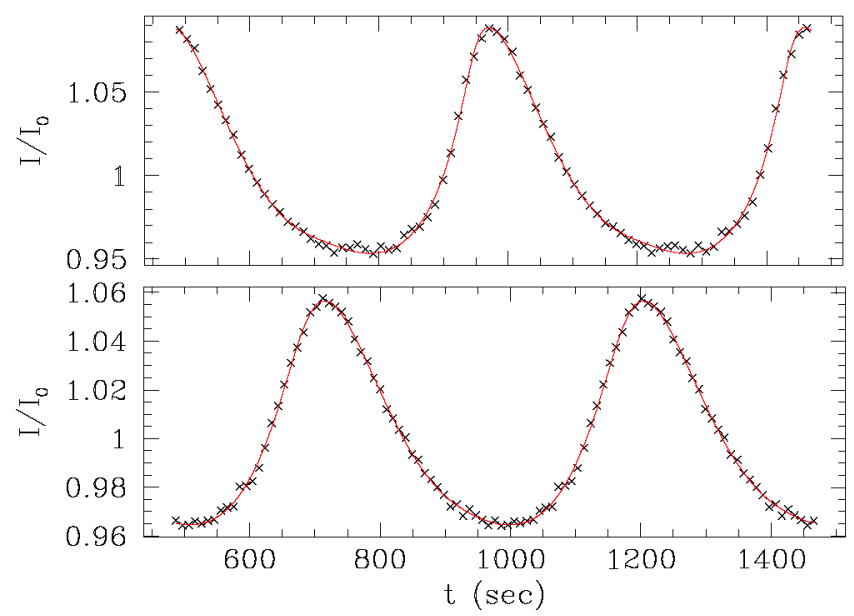

FIGURE 8. Nonlinear light curve fits to the nearly mono-periodic pulsator PG1351+489. The upper panel is a fit using data from a WET run in 1995 and the lower panel is a fit with data from 2004. These light curves have been folded at the period of the main mode, $489 \mathrm{sec}$, in order to increase $\mathrm{S} / \mathrm{N}$ and simplify the fitting.

of observations of PG1351. The only parameter which fails to lie close to or within the error bars is the amplitude of the mode. Thus, it appears that the only change which this star has undergone is a small, $\sim 20 \%$ decrease in its amplitude, which is commonly seen in these pulsators. On the other hand, the timescale $\tau_{0}$ is unchanged, indicating that the size of the convection zone has remained essentially constant. We also note that since the inclination angle, $\theta_{\mathrm{i}}$, is unchanged, this indicates that the direction of the pulsation axis has remained constant; this may be the first such "direct" evidence of this for a pulsating white dwarf.

\section{CONCLUSIONS}

While convection has traditionally been a difficulty which must be overcome, the example of Solar-type oscillations and white dwarf pulsations shows that it is also provides an opportunity for us to place constraints on it. Thus, the future promises to bring us a new understanding of convection in stellar environments.

TABLE 1. Summary of parameters derived from fits in Figures 8, 9 (Montgomery 2005a).

\begin{tabular}{rrrrrrrr}
\hline Star Name & Epoch & \multicolumn{1}{c}{$\theta_{\mathrm{i}}$} & \multicolumn{1}{c}{$\tau_{0}$} & \multicolumn{1}{c}{$N$} & \multicolumn{1}{c}{$A$} & $\ell$ & $m$ \\
\hline G29-38 & 1988 & $65.5 \pm 3.4$ & $187.4 \pm 20.3$ & $95.0 \pm 7.7$ & $0.259 \pm 0.011$ & 1 & 1 \\
PG1351 & 1995 & $57.8 \pm 1.6$ & $86.7 \pm 8.3$ & $22.7 \pm 1.3$ & $0.328 \pm 0.018$ & 1 & 0 \\
PG1351 & 2004 & $58.9 \pm 3.1$ & $89.9 \pm 3.6$ & $19.2 \pm 2.1$ & $0.257 \pm 0.021$ & 1 & 0 \\
\hline
\end{tabular}




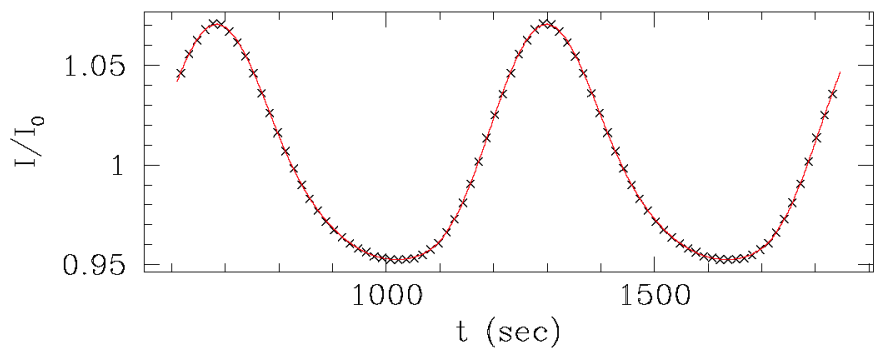

FIGURE 9. Nonlinear light curve fits to the nearly mono-periodic pulsator G29-38. The data were taken in 1988. The high $\mathrm{S} / \mathrm{N}$ of this pulse shape is the result of folding several weeks worth of data on this star at the dominant period, $615 \mathrm{sec}$.

\section{ACKNOWLEDGMENTS}

I would like to thank G. Houdek and J. Christensen-Dalsgaard for useful discussions. This research was supported in part by the National Science Foundation through grant AST-0507639.

\section{REFERENCES}

Balmforth N. J., 1992a, MNRAS, 255, 632

Balmforth N. J., 1992b, MNRAS, 255, 603

Basu S., Antia H. M., 1997, MNRAS, 287, 189

Brickhill A. J., 1991, MNRAS, 251, 673

Chaplin W. J., Houdek G., Elsworth Y., Gough D. O., Isaak G. R., New R., 2005, MNRAS, 360, 859

Christensen-Dalsgaard J., 2004, in Danesy D., ed., SOHO 14 Helio- and Asteroseismology: Towards a Golden Future, Vol. 559, p. 1

Christensen-Dalsgaard J., Gough D. O., Thompson M. J., 1991, ApJ, 378, 413

Cowling T. G., 1941, MNRAS, 101, 367

Dupret M.-A., Grigahcène A., Garrido R., Gabriel M., Noels A., 2004, in Danesy D., ed., SOHO 14 Helioand Asteroseismology: Towards a Golden Future, Vol. 559, p. 207

Goldreich P., Wu Y., 1999, ApJ, 511, 904

Gough D. O., 1977, ApJ, 214, 196

Gough D. O., 1993, in Zahn J.-P., Zinn-Justin J., eds, Astrophysical fluid dynamics Linear adiabatic stellar pulsation. Elsevier Science Publishers, Amsterdam, p. 399

Houdek G., Balmforth N. J., Christensen-Dalsgaard J., Gough D. O., 1999, A\&A, 351, 582

Monteiro M. J. P. F. G., Christensen-Dalsgaard J., Thompson M. J., 2002, in Battrick B., Favata F., Roxburgh I. W., Galadi D., eds, Stellar Structure and Habitable Planet Finding, Vol. 485, p. 291

Montgomery M. H., 2005a, ApJ, 633, 1142

Montgomery M. H., 2005b, in Koester D., Moehler S., eds, Proceedings of the 14th European Workshop on White Dwarfs, ASP, San Francisco, p. 483

Montgomery M. H., 2006, Memorie della Societa Astronomica Italiana, 77, 464

Montgomery M. H., 2007, Communications in Asteroseismology, 150, 253

Tassoul M., 1980, ApJS, 43, 469

Winget D. E., 1982, PhD thesis, University of Rochester

Winget D. E., Nather R. E., Clemens J. C., Provencal J. L., et al. 1991, ApJ, 378, 326

Winget D. E., Nather R. E., Clemens J. C., Provencal J. L., et al. 1994, ApJ, 430, 839

Winget D. E., van Horn H. M., Tassoul M., Fontaine G., Hansen C. J., Carroll B. W., 1982, ApJ, 252, L65

Wu Y., 1997, PhD thesis, California Institute of Technology

Wu Y., 2001, MNRAS, 323, 248 